Ana Carolina Brandão de Campos FONSECA PINTO ${ }^{1}$ Masao IWASAKI ${ }^{1}$

Cláudia Maria de

FIGUEIREDO $^{2}$

Sílvia Renata Gaido

CORTOPASSI ${ }^{1}$

Franklin de Almeida STERMAN ${ }^{1}$

'Correspondência para:

ANA CAROLINA B. C. FONSECA PINTO

Faculdade de Medicina Veterinária e

Zootecnia, Universidade de São Paulo

Departamento de Cirurgia

Av. Orlando Marques de Paiva, 87.

anacarol@usp.br

Recebido para publicação: 28/02/2005 Aprovado para publicação: 05/04/2007

\title{
Tomografia computadorizada do tórax de cadelas portadoras de neoplasias malignas. II - Avaliação dos campos pulmonares
}

\author{
1 - Departamento de Cirurgia, Faculdade de Medicina Veterinária e \\ Zootecnia, Universidade de São Paulo, São Paulo - SP \\ 2 - Instituto Fleury, São Paulo - SP
}

\section{Resumo}

Visto a importância das neoplasias mamárias na clínica médicoveterinária e as novas perspectivas do diagnóstico por imagem, o presente trabalho visou comparar o exame tomográfico contrastado com o exame radiográfico simples dos campos pulmonares de cadelas portadoras de tumores mamários malignos. Para tanto, foram realizados exames de tomografia computadorizada contrastada da cavidade torácica de vinte fêmeas da espécie canina, de diferentes raças e idades, portadoras de neoplasias mamárias malignas encaminhadas ao Serviço de Diagnóstico por Imagem do Hospital Veterinário da Faculdade de Medicina Veterinária e Zootecnia da Universidade de São Paulo pelos Serviços de Obstetrícia e Ginecologia e de Cirurgia de Pequenos Animais da mesma instituição. Concluiu-se que, o exame tomográfico se mostrou um importante complemento do exame radiográfico na pesquisa de metástases de neoplasias mamárias em cadelas no que se refere à avaliação do interstício pulmonar.
Palvras-chave:

Tomografia computadorizada. Pulmão. Neoplasia mamária. Cadelas.

\section{Introdução}

O exame radiográfico ainda é a modalidade diagnóstica de eleição para a avaliação inicial da cavidade torácica de pequenos animais ${ }^{1,2,3}$. Por permitir um estudo conjunto dos campos pulmonares, estruturas mediastinais, pleuras, espaço pleural, arcabouço ósseo e partes moles da parede torácica, o exame radiográfico tem sido o exame de escolha para a pesquisa de metástases no tórax ${ }^{4,5}$, para tanto, segundo Miles et al. ${ }^{6}$, deve ser realizado precocemente nos animais com neoplasias primárias.

Contudo, por meio do exame radiográfico, até $13 \%$ de falsos negativos têm sido apontados quando da pesquisa de nódulos pulmonares em cães ${ }^{5}$. Lang et al. ${ }^{7}$ advertem que vários fatores interferem na detecção de massas pulmonares ressaltando os inerentes às formações pulmonares, além daqueles relacionados à técnica radiográfica e os ligados à interpretação dos exames. Eles, assim como Forrest ${ }^{8}$, acreditam que quando a análise dos filmes for realizada por apenas um leitor, três projeções radiográficas devam ser obtidas a fim de aumentar a acurácia da análise. Barthez et al. ${ }^{9}$, todavia, recomendam duas projeções radiográficas perpendiculares entre si para a pesquisa de metástases pulmonares e, em casos duvidosos até três projeções. Para Jakovljevic e Morrison ${ }^{10}$ e Rogers ${ }^{11}$ a visibilização da lesão metastática pelo exame radiográfico depende da qualidade do equipamento radiográfico, do tamanho das formações neoplásicas, do número de nódulos, da sobreposição e somação de nódulos e de sua localização no pulmão. Apesar dos aspectos de nódulos ou de massas intersticiais sólidos, múltiplos e bem definidos serem os achados radiográficos mais comuns na doença pulmonar metastática 5,10,11,12,13,14, também são observados outros padrões como nódulos pulmonares solitários, nódulos cavitários, consolidação alveolar lobar ${ }^{10,11,15,16}$, nódulos mal definidos e aumento da radiopacidade intersticial difuso 
(linear / rendilhado) 10,11,12,13,14,15,16.

Sagel e Glaizer ${ }^{17}$ e Schwarz e Tidwell ${ }^{3}$ prelecionam que metástases hematógenas apresentam freqüentemente localizações periféricas e sub-pleurais, tornando sua evidenciação no exame radiográfico mais dificultosa; o que não ocorre no exame tomográfico, conforme Schwarz e Tidwell ${ }^{3}$. Já, Rogers ${ }^{11}$ informa que os nódulos pulmonares devem ter de 6 a 10 milímetros $(\mathrm{mm})$ de diâmetro para serem identificados radiograficamente. Jakovljevic e Morrison ${ }^{10}$, por sua vez, destacam que na periferia dos pulmões, os vasos pulmonares são praticamente imperceptíveis, assim um nódulo de radiopacidade água medindo de 2 a $5 \mathrm{~mm}$, só pode ser identificado quando as radiografias são de ótima qualidade. Nódulos pulmonares na região hilar ${ }^{11,18}$, em tórax com efusão pleural ${ }^{18,19}$, efusão nos recessos para-espinal e costo-diafragmático ${ }^{11}$, podem não ser evidenciados pelo exame radiográfico. Berry, Love e Thrall ${ }^{20} \mathrm{e}$ Schwarz e Tidwell ${ }^{3}$ relatam que nódulos menores que $6 \mathrm{~mm}$, não calcificados, são improváveis de serem vistos em radiografias de tórax, ao passo que por meio do exame tomográfico nódulos pequenos de 3 a $6 \mathrm{~mm}$, segundo Schwarz e Tidwell ${ }^{3}$, e menores, de 2 a $3 \mathrm{~mm}$, de acordo com Widmer ${ }^{21}$ podem ser identificados. Assim, em comparação ao exame radiográfico, a tomografia computadorizada consegue detectar muito mais precocemente a presença de nódulos pulmonares, porquanto, não ocorre a superposição de estruturas anatômicas como vasos sangüíneos, costelas, pleura, mediastino e diafragma ${ }^{22,23}$.

Relata-se, de forma pouco freqüente, a possibilidade de acometimento do sistema linfático pulmonar por células metastáticas de neoplasias altamente malignas, invasivas e sistêmicas (linfangite carcinomatosa). Essa forma tem sido descrita em cães, associada a carcinomas mamários ${ }^{4,5,10,15,24,25}$ e o exame tomográfico é considerado mais acurado que o exame radiográfico para o diagnóstico da linfangite carcinomatosa ${ }^{26,27}$. Pelo exame tomográfico de alta resolução ela pode ser observada como nódulos perilinfáticos que predominam nas superfícies pleurais, ao redor de grandes vasos e brônquios, nos septos interlobulares e em regiões centrilobulares ${ }^{23}$.

Feeney, Fletcher e Haardy ${ }^{28}$ asseveram que o exame radiográfico convencional é uma excelente opção para localizar alterações envolvendo estruturas da cavidade torácica, e que, as outras modalidades diagnósticas ainda não superam a radiografia simples quando se cogita a combinação de sensibilidade, especificidade e economia. Afirmam, ainda, que apesar de o exame de tomografia computadorizada poder detectar lesões neoplásicas metastáticas e infiltrados pulmonares mais precocemente que o exame radiográfico, além de fornecer avaliação anatômica mais detalhada em relação às estruturas do mediastino, espaço pleural e diafragma, para estes autores, o custo benefício do exame impede o seu uso em larga escala para este propósito. Além de que, como colocam os autores, a melhora no diagnóstico em comparação às técnicas radiográficas simples é, em geral, pequena. De forma similar, Davis ${ }^{29}$ afirmam que na medicina o exame tomográfico não será realizado em todos os casos devido ao custo-benefício e à exposição à radiação. Davis $^{29}$ acredita que a tomografia de tórax deva ser feita quando as radiografias torácicas mostrarem apenas um nódulo, quando o achado não for conclusivo e em pacientes portadores de neoplasia malignas extratorácicas com radiografias torácicas normais.

Em medicina, a tomografia computadorizada é considerada a modalidade de imagem mais sensível para a detecção de metástases pulmonares ${ }^{3,29,30,31}$. Apesar do método possibilitar a detecção precoce de nódulos pulmonares, a distinção entre lesões malignas e benignas ainda é considerada um problema ${ }^{22,23}$.

A atelectasia decúbito dependente e a estase gravitacional requerem atenção especial pela possibilidade de interferir na avaliação da imagem dos campos pulmonares $3,4,21$. Outro fator que pode ser responsável pela ocorrência de atelectasias, 
por meio de mecanismos ainda não esclarecidos, é o procedimento anestésico ${ }^{32}$. Suter ${ }^{4}$ afirma que em pacientes anestesiados o colapso do lobo pulmonar decúbitodependente pode ocorrer precocemente com apenas 3 a 5 minutos de decúbito. Cardoso e Ribeiro ${ }^{33}$, Nunn $^{34}$ e West ${ }^{35}$ relatam que a utilização de oxigênio em altas concentrações, utilizada na ventilação de pacientes, também, pode ser responsável por formação de atelectasia em menos de uma hora.

Este trabalho teve por objetivo comparar o exame tomográfico contrastado com o exame radiográfico simples na avaliação dos campos pulmonares de cadelas portadoras de neoplasias mamárias malignas.

\section{Material e Método}

Foram realizados exames radiográficos e de tomografia computadorizada contrastada da cavidade torácica de vinte fêmeas da espécie canina, de diferentes raças e idades, portadoras de neoplasias mamárias malignas atendidas pelos Serviços de Obstetrícia e Ginecologia e de Cirurgia de Pequenos Animais do Hospital Veterinário da Faculdade de Medicina Veterinária e Zootecnia da Universidade de São Paulo e, encaminhadas ao Serviço de Diagnóstico por Imagem do Hospital Veterinário da Faculdade de Medicina Veterinária e Zootecnia da Universidade de São Paulo. Constaram do grupo experimental somente animais que apresentaram radiografias do tórax sem alterações dignas de nota, com alterações radiográficas suspeitas, ou com coleções líquidas pleurais que impedissem a avaliação adequada da cavidade torácica.

Para realização do exame radiográfico foi utilizado aparelho de radiodiagnóstico, marca RAY-TEC, de $500 \mathrm{~mA}$ e $125 \mathrm{kV}$, modelo RT 500/125, comando com sistema microprocessado, gerador retificado com silício em onda completa, mesa radiológica com grade antidifusora e Bucky tipo recipromatic, ampola de Raios-X (Toshiba) de anodo giratório. Os filmes radiográficos utilizados, TMS-1 e MXG/PLUS (Kodak
Bras. Com. e Ind. Ltda), de tamanhos 24x30 $\mathrm{cm}$ e $30 \times 40 \mathrm{~cm}$, colocados em chassi metálico portando telas intensificadoras CRONEX HI plus (Du Pont Nemaves e Co.), foram selecionados de acordo com o porte do animal. Os filmes foram revelados e fixados em Processadora Automática RPX-OMAT Processor (Eastman Kodak Company), após identificação luminosa apropriada. Os animais foram posicionados em decúbito lateral direito, no caso da projeção laterolateral direita, em decúbito lateral esquerdo, no caso da projeção laterolateral esquerda e em decúbito dorsal, no caso da projeção ventrodorsal.

Para realização do exame tomográfico foi utilizado equipamento da marca GE modelo CT-MAX 640 (General Electric) de terceira geração. As imagens foram fotografadas em câmera multiformato MFC640 (General Electric) em filmes de marcas MN NIF Agfa IBF Medix (IBF Indústria Brasileira de Filmes) e Kodak Ektanscan M (Kodak Brasileira Com. Ind. Ltda) tamanho $35 \mathrm{x} 43 \mathrm{~cm}$, os quais foram revelados e fixados em Processadora Automática RPX-OMAT Processor (Eastman Kodak Company). Os vinte animais selecionados neste estudo foram então submetidos à anestesia geral para possibilitar os posicionamentos desejados, bem como, a imobilidade necessária para a realização da seqüência de cortes tomográficos. Para tanto, os cães foram prétratados com 0,05 a $0,1 \mathrm{mg} / \mathrm{kg}$ de acepromazina (Acepran ${ }^{\circledR}$ a $0,2 \%$ - Univet S.A.) e 3,0 a $5,0 \mathrm{mg} / \mathrm{kg}$ de meperidina (Dolantina-Cristália, São Paulo, SP) por meio da via intramuscular. Decorridos 20 minutos, a veia cefálica foi cateterizada e a indução da anestesia foi realizada através da administração de propofol (Diprivan Zeneca, São Paulo, SP) na dose de 5,0 mg/ $\mathrm{kg}$. Uma vez que os animais apresentaram relaxamento da região mandibular e ausência do reflexo laringotraqueal, realizou-se a intubação endotraqueal com sonda de diâmetro adequado a qual foi conectada ao circuito circular do aparelho de anestesia (Samurai III - Takaoka, São Paulo, SP). Os 
cães receberam isofluorano (Isoforine Cristália, São Paulo, SP) em oxigênio a 100\% em concentração adequada para permitir a manutenção do $2^{\circ} / 3^{\circ}$ plano de anestesia. Durante todo o procedimento, realizou-se a monitoração da freqüência e ritmo cardíaco (Sistema de Monitoração DX2010 - Dixtal, São Paulo, SP), freqüência respiratória e pressão arterial sistêmica não invasiva (Sistema de Monitoração DX2010 - Dixtal, São Paulo, SP). Os pacientes foram então posicionados em decúbito esternal com os membros torácicos tracionados cranialmente, evitando a superposição com a cavidade torácica. Os cortes tranversais se iniciaram na entrada do tórax e se estenderam até a porção mais caudal dos lobos pulmonares caudais.

$\mathrm{O}$ ajuste de técnica no aparelho de tomografia foi de $120 \mathrm{kVp}$ e 22 a $77 \mathrm{~mA}$, $\operatorname{com} 3$ a 4,8 segundos de tempo de aquisição. A espessura dos cortes foi de 5 ou $10 \mathrm{~mm}$ com incremento de 5 ou $10 \mathrm{~mm}$ entre os cortes, dependendo do porte do paciente, buscando-se atingir um número médio de 30 cortes. As imagens foram obtidas após injeção intravenosa de contraste iodado hidrossolúvel não iônico Omnipaque $300^{\circledR}$ (Searle LTDA. Para Nycomed Imaging AS) no volume aproximado de $2 \mathrm{ml} / \mathrm{kg}$ de peso, sendo dois terços da dose administrados em bolo e o complemento sob infusão contínua (gotejamento sem ocorrência de fio contínuo). Em um dos animais, com peso de $40 \mathrm{~kg}$, utilizou-se o contraste iodado hidrossolúvel iônico Hypaque ${ }^{\circledR}$ 50\% (Ind Química e Farmac. Schering-Plough). As imagens adquiridas foram fotografadas em câmera multiformato MFC640 (General Electric), com seleções de janela e nível que permitissem adequada avaliação de campos pulmonares.

A análise dos exames radiográficos e tomográficos dos animais estudados foi realizada por médico veterinário seguindo protocolo previamente elaborado que levou em consideração presença ou ausência de alterações nos pulmões, segundo classificação por quadros pulmonares (alveolar, intersticial, brônquico, vascular e misto) como proposto por Myer ${ }^{36,37,38}$. A leitura dos exames tomográficos foi repetida posteriormente em conjunto com médico radiologista sendo, desta forma, realizado um consenso sobre as possíveis alterações observadas.

\section{Resultados}

O cães que participaram desta pesquisa apresentaram características corpóreas bastante distintas assim, a distribuição dos animais estudados nesta pesquisa de acordo com à raça foi de:

Tabela 1 - Freqüências e respectivas porcentagens dos 20 cadelas portadores de neoplasias mamárias malignas que foram submetidos à avaliação radiográfica etomográfica da cavidadetorácica para pesquisademetástases, segundo os achados radiográficos em campos pulmonares, São Paulo-2001/2003

\begin{tabular}{lcc}
\hline \multicolumn{1}{c}{ Achados Radiográficos Pulmão } & Número de Cadelas & Porcentagem \% \\
\hline NDN $^{1}$ & 16 & 80 \\
Quadro intersticial (difuso) & 2 & 10 \\
Quadro intersticial (nódulo suspeito) & 1 & 5 \\
Quadro misto (intersticial e vascular) & 1 & 5 \\
\hline Total & 20 & 100 \\
\hline
\end{tabular}

${ }^{1} \mathrm{NDN}=$ exames sem evidências de alterações radiográficas dignas de nota

Tabela 2 - Freqüências e respectivas porcentagens dos 20 cadelas portadores de neoplasias mamárias malignas que foram submetidos à avaliação radiográfica e tomográfica da cavidade torácica para pesquisa de metástases, segundo os quadros pulmonares observados ao exame tomográfico, São Paulo- 2001/2003

\begin{tabular}{lcc}
\hline \multicolumn{1}{c}{ Achados Tomográficos Pulmão } & $\mathrm{N}^{\circ}$ Cadelas & Porcentagem $\%$ \\
\hline Quadro alveolar & 5 & 25 \\
Quadro brônquico & 6 & 30 \\
Quadro misto & 9 & 45 \\
\hline Total & 20 & 100 \\
\hline
\end{tabular}


cinco cães da raça Dachushund (25\%), três $(15 \%)$ da raça Cocker, dois (10\%) Poodles, um $(5 \%)$ da raça Terrier brasileiro (Fox Paulistinha), um (5\%) Pinscher, um (5\%) Mastin Napolitano, um (5\%) da raça Pastor Alemão e $6(30 \%)$ sem definição racial (SRD). As idades dessas cadelas variaram de quatro a catorze anos, sendo que 50\% dos animais tinham idades entre 10 e 14 anos, $35 \%$ variaram entre 7 e 9 anos, e apenas $15 \%$ tinham idades entre 4 e 6 anos. Quanto ao peso, observou-se uma variação entre 4,5 e $45 \mathrm{~kg}$, com $55 \%$ dos animais pesando até $10,9 \mathrm{~kg}, 20 \%$ pesando entre 11 e 20,9kg, $15 \%$ das cadelas pesando entre 21 e $30,9 \mathrm{~kg}$ e apenas dois animais pesando mais de $31 \mathrm{~kg}(10 \%)$. Nas tabelas de 1 a 4 estão apresentados os achados radiográficos e tomográficos.

\section{Discussão}

Os exames tomográficos dos campos pulmonares permitiram o achado

Tabela 3 - Freqüências e respectivas porcentagens dos 20 cadelas portadores de neoplasias mamárias malignas que foram submetidos à avaliação radiográfica e tomográfica da cavidade torácica para pesquisa de metástases, segundo o número de vezes que foram observados os quadros pulmonares ao exame tomográfico, São Paulo- 2001/2003

\begin{tabular}{lcc}
\hline \multicolumn{1}{c}{ Achados Tomográficos Pulmão } & Número de Observações ${ }^{1}$ & Porcentagem $\%$ \\
\hline Quadro alveolar & 12 & 38,71 \\
Quadro intersticial & 5 & 16,13 \\
Quadro brônquico & 13 & 41,93 \\
Quadro vascular & 1 & 3,23 \\
\hline Total & 31 & 100 \\
\hline
\end{tabular}

'Em alguns pacientes foram observados mais de um tipo de quadro pulmonar (45\% de quadro misto).

Tabela 4 - Freqüências e respectivas porcentagens dos 20 cadelas portadores de neoplasias mamárias malignas que foram submetidos à avaliação radiográfica e tomográfica da cavidade torácica para pesquisa de metástases, segundo a presença ou ausência de nódulos pulmonares ao exame tomográfico, São Paulo- 2001/2003

\begin{tabular}{lcc}
\hline Nódulos Pulmonares & Número de Cadelas & Porcentagem $\%$ \\
\hline Ausência & 15 & 75 \\
Presença & 5 & 25 \\
\hline Total & 20 & 100 \\
\hline
\end{tabular}

\begin{tabular}{|c|c|}
\hline Cadelas & Avaliação tomográfica dos pulmões \\
\hline 1 & quadro alveolar \\
\hline 2 & quadro alveolar \\
\hline 3 & quadro intersticial (2 pequenos nódulos $/ 2 \mathrm{~mm})+$ quadro brônquico(brônquios $>$ vasos) \\
\hline 4 & quadro brônquico (brônquios $>$ vasos) \\
\hline 5 & quadro alveolar + quadro intersticial + quadro vascular \\
\hline 6 & quadro brônquico (brônquios>vasos) \\
\hline 7 & quadro brônquico (brônquios>vasos) \\
\hline 8 & quadro alveolar+ quadro brônquico (alguns brônquios>vasos) \\
\hline 9 & quadro brônquico (brônquio $>$ vasos) \\
\hline 10 & $\begin{array}{c}\text { quadro alveolar. + quadro brônquico (brônquios }>\text { vasos) }+ \text { quadro intersticial (nódulo } \\
\text { subpleural } 1 \mathrm{~mm}+\text { opacidademal definida) }\end{array}$ \\
\hline 11 & quadro alveolar + quadro brônquico (brônquios > vasos) \\
\hline 12 & quadro alveolar + quadro intersticial (nódulo $5 \mathrm{~mm}$ ) \\
\hline 13 & quadro brônquico (brônquios>vasos) + quadro intersticial (nódulos 1 e 2 mm) \\
\hline 14 & quadro alveolar+quadro brônquico (alguns brônquios>vasos) \\
\hline 15 & quadro alveolar \\
\hline 16 & quadro brônquico (brônquios.>vasos) \\
\hline 17 & quadro alveolar \\
\hline 18 & quadro alveolar \\
\hline 19 & quadro brônquico (brônquios > vasos) \\
\hline 20 & quadro alveolar + quadro brônquico (brônquios $>$ vasos) \\
\hline
\end{tabular}

Quadro 1- Dados detalhados referentes aos achados tomográficos dos campos pulmonares dos vinte animais pertencentes ao estudo 
de delicadas estruturas que os compõe, de forma que, não possíveis de serem avaliadas ao exame radiográfico, tais como: discretas desproporções entre os diâmetros brônquicos e vasculares (Figura 4), espessamentos de septos interlobulares (Figura 2), visibilização mais proeminente de pontos centrolobulares (Figura 2) e de nódulos pulmonares pequenos medindo até cinco milímetros (Figura 3). Os nódulos pulmonares observados ao exame tomográfico em $25 \%$ dos animais

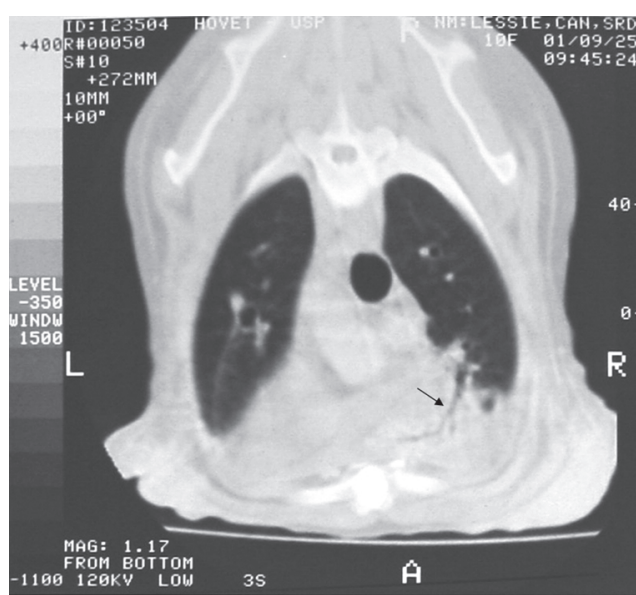

Figura 1 - Corte transversal dos lobos pulmonares craniais com $10 \mathrm{~mm}$ de espessura e seleção de janela e nível que permite adequada avaliação de campos pulmonares. Imagem representando quadro alveolar com presença de broncograma aéreo (seta) em região ventra de lobo cranial direito. Possivelmente resultante da associação de retração pulmonar decorrente da presença de líquido livre no espaço pleural aos dos efeitos da anestesia e do decúbito. Cadela SRD com10 anos de idade (animal no10)

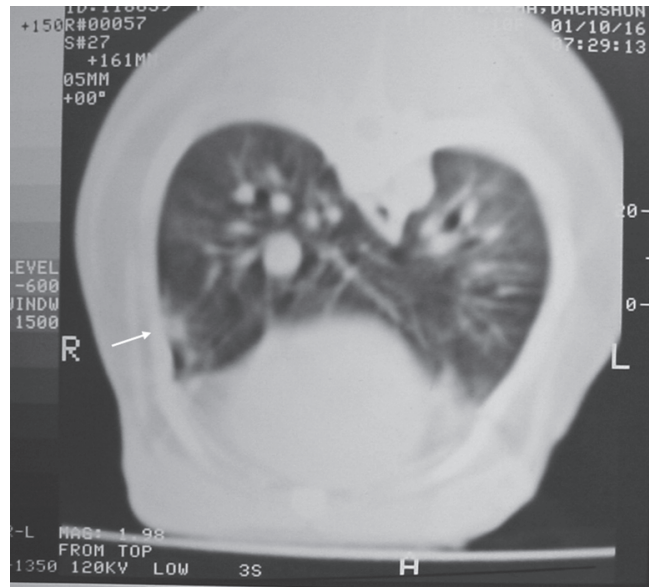

Figura 3 - Corte transversal dos lobos pulmonares caudais com $5 \mathrm{~mm}$ deespessura e seleção de janela e nível que permite adequada avaliação de campos pulmonares. Imagem representando quadro intersticia nodular, nódulo de $5 \mathrm{~mm}$ de diâmetro em região ventral de lobo cauda direito (seta). Cadela Dachshund com 9 anos de idade (animal $n^{\circ} 12$ ) estudados, não puderam ser visibilizados nas radiografias indicando que a tomografia computadorizada foi superior ao exame radiográfico convencional para detecção de nódulos pulmonares como já afirmavam Conces et al..$^{30}$, De Haan, Papageorges, $\mathrm{Kraft}^{31}$, Heitzman ${ }^{39}$, Sagel e Glazer ${ }^{17}$, Schwarz e Tidwell ${ }^{3}$ e Siegelman et al. ${ }^{40}$. O nódulo suspeito observado ao exame radiográfico do animal 20 , não foi identificado ao exame tomográfico e, portanto, foi considerado um falso positivo já que a tomografia de tórax é considerada

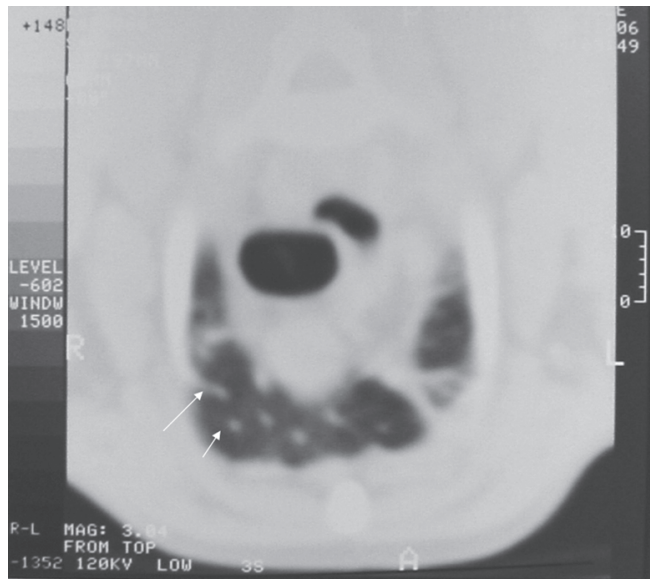

Figura 2 - Corte transversal dos lobos pulmonares craniais com 5mm de espessura e seleção de janela enível que permite adequada avaliação de campos pulmonares. Imagem representando quadro intersticial difuso com espessamento dos septos interlobulares (seta maior) e proeminência dos pontos centro lobulares (seta menor), característico de disseminação linfática e sugestivo de linfangite. Cadela Poodle com 11 anos de idade (animal n5)

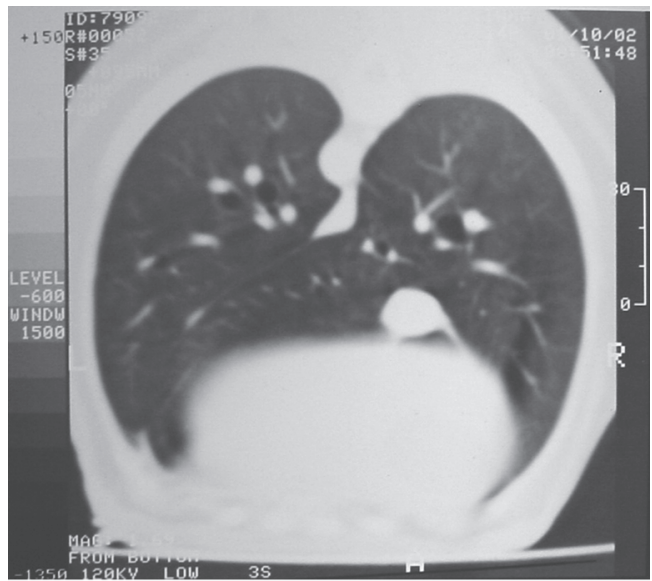

Figura 4 - Corte transversal dos lobos pulmonares caudais com $5 \mathrm{~mm}$ de espessura e seleção de janela e nível que permite adequada avaliação decampos pulmonares. Imagem representando quadro brônquico com diâmetro dos brônquios maiores que o diâmetro dos vasos correspondentes. Cadela sem raça definida com 14 anos de idade (animal $n^{\circ}$ ) 
o método de imagem mais sensível para pesquisa de nódulos pulmonares segundo Conces et al..$^{30}$, De Haan, Papageorges, $\mathrm{Kraft}^{31}$ e Schwarz e Tidwell ${ }^{3}$. Vale ressaltar que apesar de extremamente sensível para a detecção de nódulos pulmonares, o exame tomográfico tem uma baixa especificidade para diferenciação desses nódulos como sendo metastáticos ${ }^{22,23}$, granulomatosos ou gânglios linfáticos intrapulmonares, sendo necessário, portanto, outros exames para definir o diagnóstico. A visibilização de quadro intersticial difuso caracterizada pelo exame tomográfico como espessamento de septos interlobulares e pronunciamento de pontos centrolobulares como foi identificado no animal de número 5 (Figura 2) é de extrema importância tendo em vista que ele representa o comprometimento dos linfáticos pulmonares ${ }^{23}$. Essas alterações que por vezes não são indentificadas ao exame radiográfico ${ }^{26,27}$ podem representar um quadro de linfangite carcinomatosa, a qual tem sido associada aos carcinomas mamários em cadelas $4,5,10,15,24,25$. No caso desta paciente, foi descrito um quadro intersticial discreto ao exame radiográfico acometendo especialmente lobos caudais ao passo que, o quadro observado ao exame tomográfico era evidente e acometia principalmente lobos craniais. Deste modo, o quadro radiográfico visibilizado nesta fêmea de 11 anos poderia estar relacionado a outros aspectos como: radiografia expiratória ${ }^{20,41,42}$, aumento da radiopacidade pulmonar devido à idade ${ }^{4,16,41}$, ou a obesidade ${ }^{16}$.

Observou-se com grande freqüência a presença de quadro alveolar (Figura 1) identificado como atelectasia e/ou estase (60\% dos animais - Quadro 1) e atribuiu-se à ocorrência desse fato à realização do exame sob anestesia geral com utilização de oxigênio a 100\%, como fluxo diluente ${ }^{33,34,35}$, e ao tempo de duração do exame com o animal permanecendo sobre o mesmo decúbito ${ }^{3,21,43}$. A presença dessas densificações, visibilizadas em regiões ventrais dos lobos pulmonares, visto que o exame foi realizado em decúbito ventral, podem causar prejuízos na avaliação do parênquima pulmonar para pesquisa de nódulos. Desta feita, tendo em vista que a contenção química do paciente é imprescindível para realização do exame tomográfico seria conveniente a adequação do procedimento anestésico a fim de reduzir seus efeitos na formação das atelectasias. A utilização de uma menor fração inspirada de oxigênio bem como o emprego da ventilação com pressão positiva intermitente, e o uso da pressão positiva no final da expiração são elementos que poderiam prevenir a ocorrência de atelectasias tão acentuadas e tão precoces, melhorando a qualidade da imagem tomográfica dos campos pulmonares e assim incrementando sua avaliação.

Também foi com grande freqüência, em $41,93 \%$ das observações, que detectamos a presença de quadro brônquico onde foram visibilizados brônquios com diâmetro maior do que o dos vasos correspondentes, porém com paredes delicadas (Figura 4). Desta forma, apesar de Schwarz e Tidwell ${ }^{3}$ referirem que a imagem normal do parênquima pulmonar tem artérias e brônquios correspondentes com aproximadamente o mesmo diâmetro, acredita-se que novos estudos devam ser realizados em pacientes sabidamente hígidos a fim de se determinar a real proporção para o diâmetro de vasos e brônquios para que não haja interpretação errônea de dilatações brônquicas.

\section{Conclusões}

Esta pesquisa permitiu concluir que o exame tomográfico mostrou-se um importante complemento do exame radiográfico na avaliação dos campos pulmonares e na pesquisa de metástases em cadelas com neoplasias mamárias malignas. O exame tomográfico mostrou-se superior ao exame radiográfico em revelar detalhes de campos pulmonares; porém, pequenos nódulos pulmonares não puderam ser interpretados como específicos de doença metastática. Por este estudo, entende-se que novas pesquisas são necessárias para que os achados do exame tomográfico do tórax de cães possam ser interpretados com maior propriedade. 


\title{
Computed tomography exam of the thorax of female dogs with malignant mammary gland tumors. II - Pulmonary parenchimal evaluation
}

\begin{abstract}
Seen the importancy of the malignant mammary gland tumors in the veterinary medicine clinic, the new perspectives of the diagnostic imaging in order to evaluate pacients that have this neoplasia, and the few information of this subject in the literature this research proposed to compare contrast computed tomographic and survey radiographic techniques in the lung's evaluation of female dogs with malignant mammary gland tumors. This research was performed at the Diagnostic Imaging Service of the Veterinary School Hospital of the Faculdade de Medicina Veterinária e Zootecnia at the University of São Paulo in twenty, from different breed and age, bitches with malignant mammary gland tumors that were examined at the Obstetric and Ginecology and Small Animal Surgery Services of the same hospital. The tomographic exam showed up as an important complement of the radiographic exam in searching for mammary gland tumor metastasis, concerning the evaluation of the pulmonary intersticium.
\end{abstract}

Key words:

Computed tomography. Lung. Mammary

\section{Referências}

1 BURK, R. L. Radiographic examination of the cardiopulmonary system. Veterinary Clinics of North America. Small Animal Practice, v. 13, n. 2, p. 241 258, May 1983.

2 ROBERTS, R.; BANKS, W. C. Radiografhic considerations in the normal and altered cardiac silhouette of the canine patient. The Southwestern Veterinarian, v. 26, n. 1, p. 11-16, 1972.

3 SCHWARZ, L. A.; TIDWELL, A. S. Alternative imaging of the lung. Clinical Techniques in Small Animal Practice, v. 14, n. 4, p. 187-206, 1999.

4 SUTER, P. F. Thoracic radiography: a text atlas of thoracic diseases of the dog and cat. Switzerland: Peter F. Suter, 1984. cap. 1, p. 1-45; cap. 11, p. 518-682.

5 SUTER, P. F. et al. Radiographic recognition of primary and metastatic pulmonary neoplasms of dogs and cats. Journal of American Veterinary Radiology Society, v. 15, n. 3, p. 3-25, 1974.

6 MILES, K. G. et al. A retrospective evaluation of the radiographic evidence of pulmonary metastatic disease on initial presentation in the dog. Veterinary Radiology, v. 31, n. 2, p. 79-82, 1990.

7 LANG, J. et al. Sensitivity of radiographic detection of lung metastases in the dog. Veterinary Radiology, $v$. 27, n. 3, p. 74-78, 1986.

8 FORREST, L. J. Radiology corner. Advantages of three view radiographic examination in instances other than metastasis. Veterinary Radiology and Ultrasound, v. 33, p. 340-341, 1992.
9 BARTHEZ, P. Y. et al. Receiver operating characteristic curve analysis of the performance of various radiographic protocols when screening dogs for pulmonary metastases. Journal of American Veterinary Medical Association, v. 204, n. 2, p. $237-$ 240, 1994.

10 JAKOVLJEVIC, S.; MORRISON, W. B. Using conventional radiography in cancer diagnosis and monitoring. In: MORRISON, W. B. Cancer in dogs and cats: medical and surgical management. Baltimore: Williams \& Wilkins, 1998. cap. 14, p. 147-165.

11 ROGERS, K. S. Techniques for identifying metastatic tumors. Veterinary Medicine, p. 231-245, 1993. (Symposium on Veterinary Oncology)

12 GAWNE-CAIN, M. L. et al. Radiographic patterns of intrathoracic disease in breast carcinoma: prognostic implications. Clinical Radiology, v. 48, p. 253-257, 1993

13 NYKAMP, S. G.; SCRIVANI, P. V.; DYKES, N. L. Radiographic signs of pulmonary disease: an alternative approach. Compendium on Continuing Education for the Practicing Veterinarian, v. 24, n. 1, p. 25-35, 2002.

14 TIEMESSEN, I. Thoracic metastases of canine mammary gland tumors. A radiographic study. Veterinary Radiology, v. 30, n. 6, p. 249-252, 1989.

15 BRODEY, R. S.; GOLDSCHMIDT, M. H.; ROSZEL, J. R. Canine mammary gland neoplasms. Journal of the American Animal Hospital Association, v. 19, p. 61-90, Jan./ Feb. 1983.

16 LAMB, C. R. The canine and feline lung. In: THRALL, D. E. Textbook of veterinary diagnostic radiology. 4 . 
ed. Philadelphia: W. B. Saunders, 2002. cap. 34, p. 431-449

17 SAGEL, S. S.; GLAZER, H. S. Lung, pleura, chest wall. In: LEE, J. K. T.; SAGEL, S. S.; STANLEY, R. J. (Ed.). Computed body tomography with MRI correlation. New York: Raven Press, 1989. p. 295385.

18 BURK, R. L. Computed tomography of thoracic diseases in dogs. Journal of American Veterinary Medicine Association, v. 199, n. 5, p. 617-621, 1991.

19 THRALL, D. E. The pleural space. In: Textbook of veterinary diagnostic radiology. $\overline{4 . \mathrm{ed}}$. Philadelphia: W. B. Saunders, 2002. cap. 31, p. 390401.

20 BERRY, C. R.; LOVE, N. E.; THRALL, D. E. Interpretation paradigms for the small animal thorax. In: THRALL, D. E. Textbook of veterinary diagnostic radiology. 4. ed. Philadelphia: W. B. Saunders, 2002. cap. 25, p. 307-322.

21 WIDMER, W. R. Alternate imaging for the diagnosis of cancer. In: MORRISON, W. B. Cancer in dogs and cats: medical and surgical management. Baltimore: Williams \& Wilkins, 1998. cap. 16, p.187-213.

22 BURGENER, F. A.; KORMANO, M. Diagnóstico diferencial em tomografía computadorizada. Rio de Janeiro: Revinter, 1998. p. 184-214, 222-245.

23 WEBB, W. R. Doenças pulmonares. In: WEBB, W. R.; BRANT, W. E.; HELMS, C. A. Fundamentos de tomografia computadorizada do corpo. 2. ed. Rio de Janeiro: Guanabara Koogan, 2000. cap. 6, p. 88121.

24 ADAMS, W. H.; DUBEILZIG, R. Diffuse pulmonary alveolar septal metastasis from mammary carcinoma in the dog. Journal of American Veterinary Radiology Society, v. 19, p. 161-167, 1978.

25 FILDLER, I. J.; ADT, D. A.; BRODEY, R. S. Biological behavior of canine mammary neoplasms. Journal of Veterinary Medical Association, v. 151, n. 10, p. 1311$1318,1967$.

26 MATHIESON, J. R. et al. Chronic diffuse infiltrative lung disease: comparison of diagnostic accuracy of CT and chest radiography. Radiology, v. 171, p. 111-116, 1989.

27 STEIN, M. G. et al. Pulmonary lymphangitic spread of carcinoma: appeareance on CT scans. Radiology, v. 162, p. 371-375, 1987.

28 FEENEY, D. A.; FLETCHER, T. F.; HAARDY, R. M. Atlas of correlative imaging anatomy of the normal dog ultrasound and computed tomography.
Philadelphia: W. B. Saunders, 1991. p. 209-210, 335352.

29 DAVIS, S. D. CT evaluation for pulmonary matastases in patients with extrathoracic malignancy. Radiology, v. 180, p. 1-12, 1991.

30 CONCES, D. J. et al. T1N0M0 Lung cancer: evaluation with CT. Radiology, v. 170, p. 643-646, 1989.

31 DE HAAN, C. E.; PAPAGEORGES, M.; KRAFT, S. L. Radiographic diagnosis (pulmonary mass). Veterinary Radiology, v. 32, n. 2, p. 75-77, 1991.

32 WARNER, D. O.; WARNER, M. A.; RITMAN, E. L. Atelectasis and chest wall shape during anesthesia. Anestesiology, v. 85, n. 1, p. 49-59, 1996.

33 CARDOSO, P. R.; RIBEIRO, S. P. Toxicidade ao oxigênio. In: CARVALHO, C. C. R. (Ed.). Ventilação mecânica: avançado. São Paulo: Atheneu, 2001. v. 2, p. 283-290. (Clínicas brasileiras de medicina intensiva).

34 NUNN, J. F. Respiratory phisiology. 3. ed. Boston: Butterworths, 1987. p. 442-443.

35 WEST, J. B. Fisiopatologia pulmonar moderna. São Paulo: Manole, 1986. cap. 9, p. 171-186.

36 MYER, W. Radiography review: The alveolar pattern of pulmonary disease. Veterinary Radiology. v. 20, n. 1, p. 10-14, 1979.

37 MYER, W. Radiography review: The interstitial pattern of pulmonary disease. Veterinary Radiology. v. 21, n. 1 , p. $18-23,1980$.

38 MYER, W. Radiography review: The vascular and bronchial patterns of pulmonary disease. Veterinary Radiology. v. 21, n. 4, p. 156-160, 1980.

39 HEITZMAN, E. R. Computed tomography of the thorax: current perspectives. American Journal of Radiology, v. 136, p. 3-12, 1981.

40 SIEGELMAN, S. S. et al. Solitary pulmonary nodules: CT assessment. Radiology, v. 160, p. 307-312, 1986.

41 KEALY, J. K.; MACALLISTER, H. Diagnopstic radiology and ultrasonography of the dog and cat. 3 . ed. Philadelphia: W. B. Saunders, 2000. cap. 3, p. 149251.

42 SILVERMAN, S.; SUTER, P. F. Influence of inspiration and expiration on canine thoracic radiographs. Journal of American Veterinary Medical Association, v. 166, n. 5, p. 502-510, 1975.

43 AHLBERG, N. E. et al. A computed tomographic study of volume and X-ray attenuation of the lungs of beagles in various body positions. Veterinary Radiology, v. 26, n. 2, p. 43-47, 1985. 